\title{
Maximum Observed Excretion Rate Normalized by Weight
}

National Cancer Institute

\section{Source}

National Cancer Institute. Maximum Observed Excretion Rate Normalized by Weight. NCI

Thesaurus. Code C92398.

The maximum observed excretion rate divided by the weight. 